\title{
Periodicity of powers of intuitionistic fuzzy matrices
}

\author{
P. Murugadas ${ }^{1 *}$, G. Kannadasan ${ }^{2}$ and Riyaz Ahmad Padder ${ }^{3}$
}

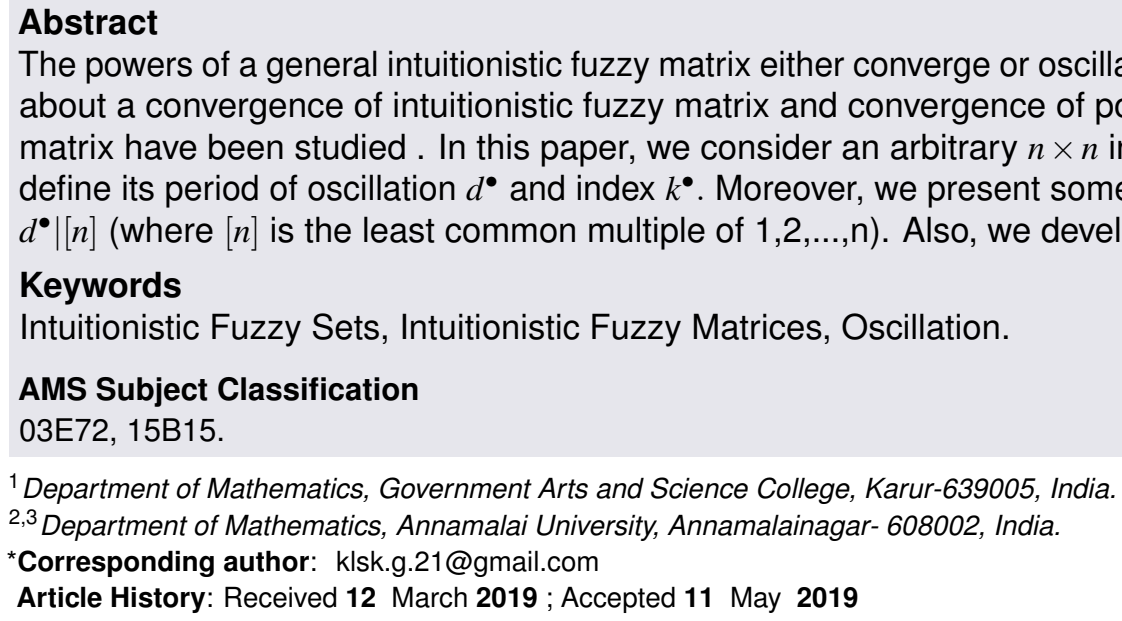

\section{Keywords}

Intuitionistic Fuzzy Sets, Intuitionistic Fuzzy Matrices, Oscillation.

\section{AMS Subject Classification} 03E72, 15B15.

${ }^{1}$ Department of Mathematics, Government Arts and Science College, Karur-639005, India.

2,3 Department of Mathematics, Annamalai University, Annamalainagar- 608002, India.

*Corresponding author: klsk.g.21@gmail.com

Article History: Received 12 March 2019 ; Accepted 11 May 2019 about a convergence of intuitionistic fuzzy matrix and convergence of powers of a transitive intuitionistic fuzzy matrix have been studied. In this paper, we consider an arbitrary $n \times n$ intuitionistic fuzzy matrix $\left(\left\langle q_{i j}, q_{i j}^{\prime}\right\rangle\right)$ and define its period of oscillation $d^{\bullet}$ and index $k^{\bullet}$. Moreover, we present some properties of $d^{\bullet}$ and $k^{\bullet}$ and show that $d^{\bullet} \mid[n]$ (where $[n]$ is the least common multiple of $1,2, \ldots, \mathrm{n}$ ). Also, we develop the algorithm to find $d^{\bullet}$ and $k^{\bullet}$.

(C)2019 MJM.

\section{Contents}

1 Introduction $\ldots \ldots \ldots \ldots \ldots \ldots \ldots \ldots \ldots \ldots \ldots \ldots, 314$

2 Preliminaries .............................. 315

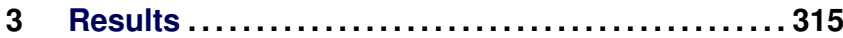

4 An algorithm to find the period of oscillation.....317

5 Conclusion ............................. 318

References .............................. 318

\section{Introduction}

Fuzzy sets introduced by Zadeh [32] has shown meaningful applications in many fields of study. The idea of fuzzy set is welcome because it handles uncertainty and vagueness which classical set could not address. However, it often falls short of the expected standard when describing the neutral state. As a result, a new concept namely Intuitionistic Fuzzy Set (IFS) was introduced by Atanassov [5] and he has represented it as $A=\left\{\left\langle x, \mu_{A}(x), v_{A}(x)\right\rangle / x \in E\right\}$ where $\mathrm{E}$ denotes a universal set, in which $\mu_{A}: E \rightarrow[0,1]$ and $v_{A}: E \rightarrow[0,1]$ denote membership and non-membership functions of $A$ respectively and its sum is less than or equal to one. In short we write the elements of IFS as $\left\langle x, x^{\prime}\right\rangle$ such that $x+x^{\prime} \leq 1$. The ideas of IFS were developed later in [6-10]. Xu and Yager [31] have developed some geometric operators based on IFSs.

A Fuzzy Matrix (FM) is a matrix with elements having values in closed interval [0,1]. Kim and Roush [33] have introduced the concept of FM. FM play a vital role in various areas, in Science and Engineering and solve the problems involving various types of uncertainties [34]. Meenakshi [17] has studied minus ordering, space ordering and schur complement of FM and block FM. Buckley [39], Ran and Liu [35] and Gregory et. al, [38] after applying max-min operation on fuzzy matrix have found only two results, either the fuzzy matrix convergences to idempotent matrix or oscillates to finite period. Hashimoto [37] has studied the convergence of power of a fuzzy transitive matrix. Lur et. al, [36] have studied the powers of convergence of FMs. Many authors have further, extended this study and addressed themselves to the discussion of special matrices such as symmetric fuzzy matrices, nilpotent fuzzy matrices, controllable fuzzy matrices, max-min transitive fuzzy matrices, etc., [40, 42, 44]. The periodicity of these special matrices is 1 or 2 [44]. Li [43] has studied periodicity and index of fuzzy matrices in the general case. In [41] Fan has proved that the periodicity of a fuzzy matrix is the least common multiple (l.c.m.) of periodicity of its cut matrices, and the index of a fuzzy matrix is not greater than the maximum index of its cut matrices. Liua and Ji [46] have discussed the periodicity of square fuzzy matrices based on minimal strong components. The notion Intuitionistic Fuzzy Matrix (IFM) was introduced by Atanssov [3]. The index matrix representation of the intuitionistic fuzzy graphs has been studied in [4]. Pal et. al, [24], Meenakshi and Gandhimathi [16] and Sriram and Murugadas [29, 30] have studied IFM for finding intuitionistic fuzzy linear relation equation, g-inverse 
and intiuitionistic fuzzy linear transformation etc.,. Shyamal and Pal [28] have studied the distance between IFM. Bhowmik and Pal [12, 13] have examined circulant IFM and generalized IFMs. Im et al., [14] have studied determinant of square IFMs. In [11] Atanassov has studied intuitionistic fuzzy index matrix and extended intuitionistic fuzzy index matrix. Several authors [1, 2, 15, 18-20, 22, 23, 25-27] have worked on IFMs and obtained various interesting results, which are very useful in handling uncertainty problems in our daily life. The purpose of this paper is to study the periodicity of powers IFM.

\section{Preliminaries}

Throughout the paper matrix mean IFM. Atanassov has introduced the following operations on IFS. For $\left\langle x, x^{\prime}\right\rangle,\left\langle y, y^{\prime}\right\rangle \in \operatorname{IFS},\left\langle x, x^{\prime}\right\rangle \vee\left\langle y, y^{\prime}\right\rangle=\left\langle\max \{x, y\}, \min \left\{x^{\prime}, y^{\prime}\right\}\right\rangle$ and $\left\langle x, x^{\prime}\right\rangle \wedge\left\langle y, y^{\prime}\right\rangle=\left\langle\min \{x, y\}, \max \left\{x^{\prime}, y^{\prime}\right\}\right\rangle .\left\langle x, x^{\prime}\right\rangle \geq\left\langle y, y^{\prime}\right\rangle \Rightarrow$ $x \geq y$ and $x^{\prime} \leq y^{\prime}$, in this case we say $\left\langle x, x^{\prime}\right\rangle$ and $\left\langle y, y^{\prime}\right\rangle$ are comparable.

Definition 2.1. [23] Let $X=\left\{x_{1}, x_{2}, \ldots x_{m}\right\}$ be a set of alternatives and $Y=\left\{y_{1}, y_{2}, \ldots y_{n}\right\}$ be the attribute set of each element of $X$. An IFM is defined by

$A=\left(\left\langle\left(x_{i}, y_{j}\right), \mu_{A}\left(x_{i}, y_{j}\right), v_{A}\left(x_{i}, y_{j}\right)\right\rangle\right)$ for $i=1,2 \ldots m$ and $j=$ $1,2, \ldots n$, where $\mu_{A}: X \times Y \rightarrow[0,1]$ and $v_{A}: X \times Y \rightarrow[0,1]$ satisfy the condition $0 \leq \mu_{A}\left(x_{i}, y_{j}\right)+v_{A}\left(x_{i}, y_{j}\right) \leq 1$. For simplicity we denote an IFM is a matrix of pairs $A=\left(\left\langle a_{i j}, a_{i j}^{\prime}\right\rangle\right)$ of non negative real numbers satisfying $a_{i j}+a_{i j}^{\prime} \leq 1$ for all $i, j$. We denote the set of all IFM of order $m \times n$ by $\mathscr{F}_{m n}$.

Consider the IFMs, $Q=\left(\left\langle q_{i j}, q_{i j}^{\prime}\right\rangle\right)$ and $P=\left(\left\langle p_{i j}, p_{i j}^{\prime}\right\rangle\right)$. We recall some basic operation.

$Q P=\left(\bigvee_{k=1}^{n}\left(\left\langle q_{i k} \wedge p_{k j}\right), \bigwedge_{k=1}^{n}\left(q_{i k}^{\prime} \vee p_{k j}^{\prime}\right\rangle\right)\right)$

$Q^{K+1}=Q^{K} Q$, for all $k=1,2,3, \ldots$

$Q^{0}=I$, where $\mathrm{I}$ is a square unit matrix all whose diagonal entries are $\langle 1,0\rangle$ and all other are $\langle 0,1\rangle$.

$Q^{k}=\left(\left\langle q_{i j}^{k}, q_{i j}^{\prime k}\right\rangle\right)$, for all $k=1,2,3, \ldots$. Then,

$\left\langle q_{i j}, q_{i j}^{\prime}\right\rangle=\bigvee_{J_{1}=1}^{n} \bigvee_{j_{2}=1}^{n} \ldots \bigvee_{j_{k-1}=1}^{n}\left(\left\langle q_{i j_{1}}, q_{i j_{1}}^{\prime}\right\rangle \wedge\left\langle q_{j_{1} j_{2}}, q_{j_{1} j_{2}}^{\prime}\right\rangle \wedge \ldots \wedge\right.$

$\left.\left\langle q_{j_{k-i} j}, q_{j_{k-i} j}^{\prime}\right\rangle\right)$.

$Q \leq P$ iff $\left\langle q_{i j}, q_{i j}^{\prime}\right\rangle \leq\left\langle p_{i j}, p_{i j}^{\prime}\right\rangle$ for all $i, j \in\{1,2, \ldots, n\}$.

Let $I^{+}=\{x \mid x$ is a positive integer $\}$.

Remark 2.2. [30] Let $Q, S, H$ and $T$ be $n \times n$ intuitionistic fuzzy matrices. If $Q \leq S, T \leq H, \Rightarrow Q T \leq S H$,

\section{Results}

Let $\left(\left\langle q_{i j}, q_{i j}^{\prime}\right\rangle\right)$ be an arbitrary $n \times n$ intuitionistic fuzzy matrix. The number of different matrices is at most $r^{n^{2}}$ in the following sequence,

$$
\left(\left\langle q_{i j}, q_{i j}^{\prime}\right\rangle\right),\left(\left\langle q_{i j}^{2}, q_{i j}^{\prime 2}\right\rangle\right),\left(\left\langle q_{i j}^{3}, q_{i j}^{\prime 3}\right\rangle\right), \ldots,\left(\left\langle q_{i j}^{m}, q_{i j}^{\prime m}\right\rangle\right), \ldots,
$$

where $r$ is the number of all the different elements that occur in intuitionistic fuzzy matrix $\left(\left\langle q_{i j}, q_{i j}^{\prime}\right\rangle\right)$, which is clearly finite. Thus there exists $s, t \in I^{+}(s \neq t)$ such that $\left(\left\langle q_{i j}^{s}, q_{i j}^{\prime s}\right\rangle\right)=$ $\left(\left\langle q_{i j}^{t}, q_{i j}^{\prime t}\right\rangle\right)$. Let

$H=\left\{(s, t) \mid\left(\left\langle q_{i j}^{s}, q_{i j}^{\prime s}\right\rangle\right)=\left(\left\langle q_{i j}^{t}, q_{i j}^{\prime t}\right\rangle\right), s \neq t, s, t \in I^{+}\right\}$

$D=\{d|d=| s-t \mid,(s, t) \in H\}$.

Then, D has a least element $d^{\bullet}\left(d^{\bullet} \geq 1\right)$ by using the wellordering property of natural numbers.

Let

$K=\left\{k \mid\left(\left\langle q_{i j}^{k}, q_{i j}^{\prime k}\right\rangle\right)=\left(\left\langle q_{i j}^{k+d^{\bullet}}, q_{i j}^{\prime k+d^{\bullet}}\right\rangle\right), k \in Z^{+}\right\}$

By well-ordering property, $\mathrm{K}$ has a least element $k^{\bullet}\left(k^{\bullet} \geq 1\right)$.

Definition 3.1. We call $d^{\bullet}$ the period of oscillation of $\left(\left\langle q_{i j}, q_{i j}^{\prime}\right\rangle\right)$, and $k^{\bullet}$ the index of $\left(\left\langle q_{i j}, q_{i j}^{\prime}\right\rangle\right) .\left(\left\langle q_{i j}, q_{i j}^{\prime}\right\rangle\right)$ is convergent if $d^{\bullet}=1$

Lemma 3.2. Let $q \in I^{+}$. If $q \geq k^{\bullet}$, then

$\left(\left\langle q_{i j}^{q}, q_{i j}^{\prime q}\right\rangle\right)=\left(\left\langle q_{i j}^{q+m d^{\bullet}}, q_{i j}^{\prime q+m d^{\bullet}}\right\rangle\right)$ for any $m \in I^{+}$.

Proof. We will prove this lemma by induction on $m \geq 1$

For $m=1$,

since $\left(\left\langle q_{i j}^{k^{\bullet}}, q_{i j}^{\prime k^{\bullet}}\right\rangle\right)=\left(\left\langle q_{i j}^{k^{\bullet}+d^{\bullet}}, q_{i j}^{\prime k^{\bullet}+d^{\bullet}}\right\rangle\right)$, then

$\left(\left\langle q_{i j}^{q-k^{\bullet}}, q_{i j}^{\prime q-k^{\bullet}}\right\rangle\right) \times\left(\left\langle q_{i j}^{k^{\bullet}}, q_{i j}^{\prime k^{\bullet}}\right\rangle\right)$

$=\left(\left\langle q_{i j}^{q-k^{\bullet}}, q_{i j}^{\prime q-k^{\bullet}}\right\rangle\right)$

$\times\left(\left\langle q_{i j}^{k^{\bullet}+d^{\bullet}}, q_{i j}^{\prime k^{\bullet}+d^{\bullet}}\right\rangle\right)$,

and the required equality $\left(\left\langle q_{i j}^{q}, q_{i j}^{\prime q}\right\rangle\right)=\left(\left\langle q_{i j}^{k^{\bullet}+d^{\bullet}}, q_{i j}^{\prime k^{\bullet}+d^{\bullet}}\right\rangle\right)$, is obtained.

Let $\left(\left\langle q_{i j}^{q}, q_{i j}^{\prime q}\right\rangle\right)=\left(\left\langle q_{i j}^{q+(m-1) d^{\bullet}}, q_{i j}^{q q+(m-1) d^{\bullet}}\right\rangle\right)$, then

$\left(\left\langle q_{i j}^{d^{\bullet}}, q_{i j}^{\prime d^{\bullet}}\right\rangle\right) \times\left(\left\langle q_{i j}^{q}, q_{i j}^{\prime q}\right\rangle\right)=\left(\left\langle q_{i j}^{d^{\bullet}}, q_{i j}^{\prime d^{\bullet}}\right\rangle\right)$

$\times\left(\left\langle q_{i j}^{q+(m-1) d^{\bullet}}, q_{i j}^{\prime q+(m-1) d^{\bullet}}\right\rangle\right)$,

that is, $\left(\left\langle q_{i j}^{q+d^{\bullet}}, q_{i j}^{q+d^{\bullet}}\right\rangle\right)=\left(\left\langle q_{i j}^{q+m d^{\bullet}}, q_{i j}^{q+m d^{\bullet}}\right\rangle\right)$;

but $\left(\left\langle q_{i j}^{q+d^{\bullet}}, q_{i j}^{q+d^{\bullet}}\right\rangle\right)=\left(\left\langle q_{i j}^{q}, q_{i j}^{q}\right\rangle\right)$, so that

$\left(\left\langle q_{i j}^{q}, q_{i j}^{q q}\right\rangle\right)=\left(\left\langle q_{i j}^{q+m d^{\bullet}}, q_{i j}^{q+m d^{\bullet}}\right\rangle\right)$.

Theorem 3.3. If there exist $t, s \in I^{+}$such that $\left(\left\langle q_{i j}^{t}, q_{i j}^{\prime t}\right\rangle\right) \leq$ $\left(\left\langle q_{i j}^{s}, q_{i j}^{\prime s}\right\rangle\right)$, then $d^{\bullet} \mid(t-s)$, where a $\mid b$ means that a divides $b$.

Proof. (1) If $t=s$, then $t-s=0$, and $d^{\bullet} \mid 0$.

(2) If $t<s$, then $\left(\left\langle q_{i j}^{t}, q_{i j}^{\prime t}\right\rangle\right) \leq\left(\left\langle q_{i j}^{t+(s-t)}, q_{i j}^{\prime t+(s-t)}\right\rangle\right)$. Using Remark 2.2 repeatedly, we will have

$$
\left(\left\langle q_{i j}^{t}, q_{i j}^{\prime t}\right\rangle\right) \leq\left(\left\langle q_{i j}^{t+(s-t)}, q_{i j}^{\prime t+(s-t)}\right\rangle\right) \leq\left(\left\langle q_{i j}^{t+2(s-t)}, q_{i j}^{\prime t+2(s-t)}\right\rangle\right)
$$

$$
\leq \ldots \leq\left(\left\langle q_{i j}^{t+m(s-t)}, q_{i j}^{\prime t+m(s-t)}\right\rangle\right) \leq \ldots
$$

Since in the sequence

$\left(\left\langle q_{i j}^{t}, q_{i j}^{\prime t}\right\rangle\right),\left(\left\langle q_{i j}^{t+(s-t)}, q_{i j}^{\prime t+(s-t)}\right\rangle\right),\left(\left\langle q_{i j}^{t+2(s-t)}, q_{i j}^{\prime t+2(s-t)}\right\rangle\right), \ldots$, $\left(\left\langle q_{i j}^{t+m(s-t)}, q_{i j}^{\prime t+m(s-t)}\right\rangle\right), \ldots$,

the number of different IFMs is finite, there exists $k, h \in I^{+}$ $(h<k)$ such that 
$\left(\left\langle q_{i j}^{t+h(s-t)}, q_{i j}^{\prime t+h(s-t)}\right\rangle\right)=\left(\left\langle q_{i j}^{t+k(s-t)}, q_{i j}^{\prime t+k(s-t)}\right\rangle\right)$. From (2), it If follows that

$$
\left(\left\langle q_{i j}^{t+h(s-t)}, q_{i j}^{\prime t+h(s-t)}\right\rangle\right)=\left(\left\langle q_{i j}^{t+h(s-t)+(s-t)}, q_{i j}^{\prime t+h(s-t)+(s-t)}\right\rangle\right) .
$$

Let $q \in I^{+}$be such that $q \geq \max \left\{t+h(s-t), k^{\bullet}\right\}$.

Then, by (3), we will have

$$
\left(\left\langle q_{i j}^{q}, q_{i j}^{q}\right\rangle\right)=\left(\left\langle q_{i j}^{q+(s-t)}, q_{i j}^{q+(s-t)}\right\rangle\right) .
$$

On the other side, Lemma 3.2 implies that

$$
\left(\left\langle q_{i j}^{q}, q_{i j}^{\prime q}\right\rangle\right)=\left(\left\langle q_{i j}^{q+d^{\bullet}}, q_{i j}^{\prime q+d^{\bullet}}\right\rangle\right)
$$

By (4), (5) and the definition of $d^{\bullet}$, we have $d^{\bullet} \leq s-t$. From the division algorithm, it follows that there are unique integers $a$ and $c$ such that

$$
s-t=a d^{\bullet}+c \text { with } 0 \leq c<d^{\bullet}, a>0 .
$$

By Lemma 3.2, (4) and (5), we have

$$
\begin{aligned}
\left(\left\langle q_{i j}^{q+c}, q_{i j}^{q+c}\right\rangle\right) & =\left(\left\langle q_{i j}^{q+c+a d^{\bullet}}, q_{i j}^{\prime q+c+a d^{\bullet}}\right\rangle\right) \\
& =\left(\left\langle q_{i j}^{q+(s-t)}, q_{i j}^{\prime q+(s-t)}\right\rangle\right)=\left(\left\langle q_{i j}^{q}, q_{i j}^{\prime q}\right\rangle\right) .
\end{aligned}
$$

From the definition of $d^{\bullet}$ and the fact $0 \leq c<d^{\bullet}$, it follows that $c=0$, and hence

$d^{\bullet} \mid(s-t)$, so $d^{\bullet} \mid(t-s)$.

(3) If $t>s$, the proof is same as of (2).

Corollary 3.4. $\left(\left\langle q_{i j}, q_{i j}^{\prime}\right\rangle\right)$ is convergent iff there exist $t_{1}, t_{2}, t_{3}, t_{4} \in I^{+}\left(t_{1} \neq t_{2}\right)$ such that $\left(\left\langle q_{i j}^{t_{1}}, q_{i j}^{\prime t_{1}}\right\rangle\right) \leq\left(\left\langle q_{i j}^{t_{2}}, q_{i j}^{\prime t_{2}}\right\rangle\right)$, $\left(\left\langle q_{i j}^{t_{3}}, q_{i j}^{\prime t_{3}}\right\rangle\right) \leq\left(\left\langle q_{i j}^{t_{4}}, q_{i j}^{\prime t_{4}}\right\rangle\right)$ and $\left(t_{1}-t_{2}, t_{3}-t_{4}\right)=1$, where $(a, b)$ denotes the greatest common divisor of $a$ and $b$.

Proof. The part of the necessity is easy to verify. We prove the sufficiency. If $\left(\left\langle q_{i j}^{t_{1}}, q_{i j}^{\prime t_{1}}\right\rangle\right) \leq\left(\left\langle q_{i j}^{t_{2}}, q_{i j}^{\prime t_{2}}\right\rangle\right),\left(\left\langle q_{i j}^{t_{3}}, q_{i j}^{\prime t_{3}}\right\rangle\right) \leq$ $\left(\left\langle q_{i j}^{t_{4}}, q_{i j}^{\prime t_{4}}\right\rangle\right)$ and $\left(t_{1}-t_{2}, t_{3}-t_{4}\right)=1$, then by Theorem 3.3, we have

$d^{\bullet}\left|\left(t_{1}-t_{2}\right), d^{\bullet}\right|\left(t_{3}-t_{4}\right)$,

which means that $d^{\bullet} \mid 1$, so, $d^{\bullet}=1$. $\Rightarrow\left(\left\langle q_{i j}, q_{i j}^{\prime}\right\rangle\right)$ is convergent.

Theorem 3.5. Let $t, h \in I^{+}$and $t \neq h$. Then, $\left(\left\langle q_{i j}^{h}, q_{i j}^{\prime h}\right\rangle\right)=\left(\left\langle q_{i j}^{t}, q_{i j}^{\prime t}\right\rangle\right)$ iff $d^{\bullet} \mid(t-h)$, and $k^{\bullet} \leq \min \{h, t\}$

Proof. We may assume without lose of generality that $h<t$.

We first prove the necessity.

Let $\left(\left\langle q_{i j}^{h}, q_{i j}^{\prime h}\right\rangle\right)=\left(\left\langle q_{i j}^{t}, q_{i j}^{\prime t}\right\rangle\right)$.

Then $\left(\left\langle q_{i j}^{h}, q_{i j}^{\prime h}\right\rangle\right)=\left(\left\langle q_{i j}^{h+(t-h)}, q_{i j}^{\prime h+(t-h)}\right\rangle\right)$, and by Theorem 3.3, we have $d^{\bullet} \mid(t-h)$. Hence there exists a positive integer $q$ such that $t-h=q d^{\bullet}$, then

$$
\left(\left\langle q_{i j}^{h}, q_{i j}^{\prime h}\right\rangle\right)=\left(\left\langle q_{i j}^{h+q d^{\bullet}}, q_{i j}^{\prime h+q d^{\bullet}}\right\rangle\right)
$$

$$
\left(\left\langle q_{i j}^{h}, q_{i j}^{h}\right\rangle\right) \neq\left(\left\langle q_{i j}^{h+d^{\bullet}}, q_{i j}^{\prime h+d^{\bullet}}\right\rangle\right)
$$

then, using (7) repeatedly, we get

$$
\begin{aligned}
\left(\left\langle q_{i j}^{h}, q_{i j}^{\prime h}\right\rangle\right) & =\left(\left\langle q_{i j}^{h+q d^{\bullet}}, q_{i j}^{\prime h+q d^{\bullet}}\right\rangle\right) \\
& =\left(\left\langle q_{i j}^{h}, q_{i j}^{\prime h}\right\rangle\right) \times\left(\left\langle q_{i j}^{q d^{\bullet}}, q_{i j}^{\prime q d^{\bullet}}\right\rangle\right) \\
& =\left(\left\langle q_{i j}^{h+q d^{\bullet}}, q_{i j}^{\prime h+q d^{\bullet}}\right\rangle\right) \times\left(\left\langle q_{i j}^{q d^{\bullet}}, q_{i j}^{\prime q d^{\bullet}}\right\rangle\right) \\
& =\left(\left\langle q_{i j}^{h}, q_{i j}^{\prime h}\right\rangle\right) \times\left(\left\langle q_{i j}^{2 q d^{\bullet}}, q_{i j}^{\prime 2 q d^{\bullet}}\right\rangle\right) \\
& =\ldots=\left(\left\langle q_{i j}^{h}, q_{i j}^{h}\right\rangle\right) \times\left(\left\langle q_{i j}^{m q d^{\bullet}}, q_{i j}^{\prime m q d^{\bullet}}\right\rangle\right) \\
& =\left(\left\langle q_{i j}^{h+m q d^{\bullet}}, q_{i j}^{\prime h+m q d^{\bullet}}\right\rangle\right),
\end{aligned}
$$

for any $m \in I^{+}$. And

$$
\begin{aligned}
\left(\left\langle q_{i j}^{h+d^{\bullet}}, q_{i j}^{\prime h+d^{\bullet}}\right\rangle\right) & =\left(\left\langle q_{i j}^{h}, q_{i j}^{\prime h}\right\rangle\right) \times\left(\left\langle q_{i j}^{d^{\bullet}}, q_{i j}^{\prime d^{\bullet}}\right\rangle\right) \\
& =\left(\left\langle q_{i j}^{h+q d^{\bullet}}, q_{i j}^{\prime h+q d^{\bullet}}\right\rangle\right) \times\left(\left\langle q_{i j}^{q d^{\bullet}}, q_{i j}^{\prime q d^{\bullet}}\right\rangle\right) \\
& =\left(\left\langle q_{i j}^{h}, q_{i j}^{\prime h}\right\rangle\right) \times\left(\left\langle q_{i j}^{(q+1) d^{\bullet}}, q_{i j}^{\prime(q+1) d^{\bullet}}\right\rangle\right) \\
& =\ldots=\left(\left\langle q_{i j}^{h}, q_{i j}^{\prime h}\right\rangle\right) \times\left(\left\langle q_{i j}^{(m q+1) d^{\bullet}}, q_{i j}^{\prime(m q+1) d^{\bullet}}\right\rangle\right) \\
& =\left(\left\langle q_{i j}^{h+m q d^{\bullet}+d^{\bullet}}, q_{i j}^{\prime h+m q d^{\bullet}+d^{\bullet}}\right\rangle\right),
\end{aligned}
$$

for any $m \in I^{+}$.

Then (8) implies that

$$
\left(\left\langle q_{i j}^{h+m q d^{\bullet}}, q_{i j}^{\prime h+m q d^{\bullet}}\right\rangle\right) \neq\left(\left\langle q_{i j}^{h+m q d^{\bullet}+d^{\bullet}}, q_{i j}^{\prime h+m q d^{\bullet}+d^{\bullet}}\right\rangle\right)
$$

for any $m \in I^{+}$

Otherwise, take $m \in I^{+}$such that $h+m q d^{\bullet} \geq k^{\bullet}$.

Then by Lemma 3.2, we have

$\left(\left\langle q_{i j}^{h+m q d^{\bullet}}, q_{i j}^{\prime h+m q d^{\bullet}}\right\rangle\right)=\left(\left\langle q_{i j}^{h+m q d^{\bullet}+d^{\bullet}}, q_{i j}^{\prime h+m q d^{\bullet}+d^{\bullet}}\right\rangle\right)$, a contradiction to (9).

Hence, $\left(\left\langle q_{i j}^{h}, q_{i j}^{\prime h}\right\rangle\right)=\left(\left\langle q_{i j}^{h+d^{\bullet}}, q_{i j}^{\prime h+d^{\bullet}}\right\rangle\right)$, and by the definition of $k^{\bullet}$, it follows that $k^{\bullet} \leq h=\min \{h, t\}$,

Now, we prove the sufficiency.

If $d^{\bullet} \mid(t-h), k^{\bullet} \leq \min \{h, t\}$, then there exists a positive integer $q$ such that $t-h=q d^{\bullet}$. Since $h \geq k^{\bullet}$, by Lemma 3.2, we have

$$
\begin{aligned}
\left(\left\langle q_{i j}^{h}, q_{i j}^{\prime h}\right\rangle\right) & =\left(\left\langle q_{i j}^{h+q d^{\bullet}}, q_{i j}^{\prime h+q d^{\bullet}}\right\rangle\right) \\
& =\left(\left\langle q_{i j}^{h+(t-h)}, q_{i j}^{\prime h+(t-h)}\right\rangle\right) \\
& =\left(\left\langle q_{i j}^{t}, q_{i j}^{\prime t}\right\rangle\right) .
\end{aligned}
$$

Theorem 3.6. In the sequence

$\left(\left\langle q_{i j}, q_{i j}^{\prime}\right\rangle\right),\left(\left\langle q_{i j}^{2}, q_{i j}^{\prime 2}\right\rangle\right),\left(\left\langle q_{i j}^{3}, q_{i j}^{\prime 3}\right\rangle\right), \ldots$, the number of different IFMs is $k^{\bullet}+d^{\bullet}-1$. Also, the set of the $k^{\bullet}+d^{\bullet}-1$ different IFMs is

$\left\{\left(\left\langle q_{i j}, q_{i j}^{\prime}\right\rangle\right),\left(\left\langle q_{i j}^{2}, q_{i j}^{\prime 2}\right\rangle\right), \ldots,\left(\left\langle q_{i j}^{k^{\bullet}}, q_{i j}^{\prime k^{\bullet}}\right\rangle\right)\right.$ $\left.\left(\left\langle q_{i j}^{k^{\bullet}+1}, q_{i j}^{\prime k^{\bullet}+1}\right\rangle\right), \ldots,\left(\left\langle q_{i j}^{k^{\bullet}+d^{\bullet}-1}, q_{i j}^{\prime k^{\bullet}+d^{\bullet}-1}\right\rangle\right)\right\}$. 
Proof. $T=\left\{\left(\left\langle q_{i j}, q_{i j}^{\prime}\right\rangle\right),\left(\left\langle q_{i j}^{2}, q_{i j}^{\prime 2}\right\rangle\right), \ldots,\left(\left\langle q_{i j}^{k^{\bullet}}, q_{i j}^{\prime k^{\bullet}}\right\rangle\right)\right.$, $\left.\left(\left\langle q_{i j}^{k^{\bullet}+1}, q_{i j}^{\prime k^{\bullet}+1}\right\rangle\right), \ldots,\left(\left\langle q_{i j}^{k^{\bullet}+d^{\bullet}-1}, q_{i j}^{\prime k^{\bullet}+d^{\bullet}-1}\right\rangle\right)\right\}$,

$W=\left\{\left(\left\langle q_{i j}^{m}, q_{i j}^{\prime m}\right\rangle\right) \mid m \in I^{+}\right\}$.

We first show $W=T$.

Let $\left(\left\langle q_{i j}^{m}, q_{i j}^{\prime m}\right\rangle\right) \in W$.

If $m-k^{\bullet}<d^{\bullet}$, then $m<k^{\bullet}+d^{\bullet}$,

and it follows that $\left(\left\langle q_{i j}^{m}, q_{i j}^{\prime m}\right\rangle\right) \in T$.

If $m-k^{\bullet} \geq d^{\bullet}$ then $m \geq k^{\bullet}+d^{\bullet}$.

Let $t=m-\left(k^{\bullet}+d^{\bullet}\right)$, clearly $t \geq 0, m=t+k^{\bullet}+d^{\bullet}>k^{\bullet}$. We have two cases.

case (1) $t<d^{\bullet}$. Then by Lemma 3.2 we have $\left(\left\langle q_{i j}^{m}, q_{i j}^{\prime m}\right\rangle\right)=\left(\left\langle q_{i j}^{k^{\bullet}+t+d^{\bullet}}, q_{i j}^{\prime k^{\bullet}+t+d^{\bullet}}\right\rangle\right)=\left(\left\langle q_{i j}^{k^{\bullet}+t}, q_{i j}^{\prime k^{\bullet}+t}\right\rangle\right) \in T$. case (2) $t \geq d^{\bullet}$. Then there exists two positive integers $a, c$ such that $t=a d^{\bullet}+c, 0 \leq c<d^{\bullet}$, and hence

$$
\begin{aligned}
\left(\left\langle q_{i j}^{m}, q_{i j}^{\prime m}\right\rangle\right) & =\left(\left\langle q_{i j}^{k^{\bullet}+t+d^{\bullet}}, q_{i j}^{\prime k^{\bullet}+t+d^{\bullet}}\right\rangle\right) \\
& =\left(\left\langle q_{i j}^{k^{\bullet}+a d^{\bullet}+c+d^{\bullet}}, q_{i j}^{\prime k^{\bullet}+a d^{\bullet}+c+d^{\bullet}}\right\rangle\right) \\
& =\left(\left\langle q_{i j}^{\left(k^{\bullet}+c\right)+(a+1) d^{\bullet}}, q_{i j}^{\prime\left(k^{\bullet}+c\right)+(a+1) d^{\bullet}}\right\rangle\right) \\
& =\left(\left\langle q_{i j}^{k^{\bullet}+c}, q_{i j}^{\prime k^{\bullet}+c}\right\rangle\right)
\end{aligned}
$$

(by Lemma 3.2).

So $\left(\left\langle q_{i j}^{m}, q_{i j}^{\prime m}\right\rangle\right) \in T$.

Thus $W \subseteq T$, and $W=T$.

Now, we show that the number of different elements of $T$ is $k^{\bullet}+d^{\bullet}-1$.

Suppose that $t, m \in\left\{1,2, \ldots, k^{\bullet}+d^{\bullet}-1\right\}, t \neq m$ and $\left(\left\langle q_{i j}^{t}, q_{i j}^{\prime t}\right\rangle\right)=\left(\left\langle q_{i j}^{m}, q_{i j}^{\prime m}\right\rangle\right)$.

If, $t>m$, then by Theorem 3.5 we get $t>m \geq k^{\bullet}$ and $d^{\bullet} \mid(t-$ $m)$. But $t>m \geq k^{\bullet}$, so we get

$t-m \leq\left(k^{\bullet}+d^{\bullet}-1\right)-k^{\bullet}=d^{\bullet}-1$,

and $d^{\bullet}>t-m$. But, from the fact that $d^{\bullet} \mid(t-m)$, we get $d^{\bullet} \leq t-m$, a contradiction.

Theorem 3.7. (1) $d^{\bullet} \mid[n]$

(2) $\left(\left\langle q_{i j}^{n}, q_{i j}^{\prime n}\right\rangle\right) \leq\left(\left\langle q_{i j}^{n+[n]}, q_{i j}^{\prime n+[n]}\right\rangle\right)$.

Proof. We first prove (2). For $\left(\left\langle q_{i j}^{k}, q_{i j}^{\prime k}\right\rangle\right), k=1,2, \ldots$, let the $(i, j)$ entry of $\left(\left\langle q_{i j}, q_{i j}^{\prime}\right\rangle\right)$

$\left\langle q_{i j}^{n}, q_{i j}^{\prime n}\right\rangle=\left\langle q_{h_{0} h_{1}}, q_{h_{0} h_{1}}^{\prime}\right\rangle \wedge\left\langle q_{h_{1} h_{2}}, q_{h_{1} h_{2}}^{\prime}\right\rangle \wedge \ldots \wedge\left\langle q_{h_{n-1} h_{n}}, q_{h_{n-1} h_{n}}^{\prime}\right\rangle$ where $h_{0}=i$ and $h_{n}=j$. We have $h_{a}=h_{b}$ for some $a, b \in$ $\{0,1,2, \ldots, n\}(a<b)$ since $h_{i} \in\{1,2, \ldots, n\}$ for every $i=$ $0,1,2, \ldots, n$. Then

$\left\langle q_{i j}^{n}, q_{i j}^{\prime n}\right\rangle=\left\langle q_{h_{0} h_{1}}, q_{h_{0} h_{1}}^{\prime}\right\rangle$

$\wedge \ldots \wedge\left\langle q_{h_{a-1} h_{a}}, q_{h_{a-1} h_{a}}^{\prime}\right\rangle \wedge$

$\left\langle q_{h_{a} h_{a+1}}, q_{h_{a} h_{a+1}}^{\prime}\right\rangle \wedge \ldots \wedge\left\langle q_{h_{b-1} h_{a}}, q_{h_{b-1} h_{a}}^{\prime}\right\rangle \wedge\left\langle q_{h_{a} h_{b+1}}, q_{h_{a} h_{b+1}}^{\prime}\right\rangle \wedge$

$\left\langle q_{h_{b+1} h_{b+2}}, q_{h_{b+1} h_{b+2}}^{\prime}\right\rangle \wedge \ldots \wedge\left\langle q_{h_{n-1} h_{n}}, q_{h_{n-1} h_{n}}^{\prime}\right\rangle$

$=\left(\left\langle q_{h_{0} h_{1}}, q_{h_{0} h_{1}}^{\prime}\right\rangle \wedge \ldots \wedge\left\langle q_{h_{a-1} h_{a}}, q_{h_{a-1} h_{a}}^{\prime}\right\rangle\right) \wedge$

$\left(\left\langle q_{h_{a} h_{a+1}}, q_{h_{a} h_{a+1}}^{\prime}\right\rangle \wedge \ldots \wedge\left\langle q_{h_{b-1} h_{a}}, q_{h_{b-1} h_{a}}^{\prime}\right\rangle\right) \wedge\left(\left\langle q_{h_{a} h_{b+1}}, q_{h_{a} h_{b+1}}^{\prime}\right\rangle\right.$

$\left.\wedge\left\langle q_{h_{b+1} h_{b+2}}, q_{h_{b+1} h_{b+2}}^{\prime}\right\rangle \wedge \ldots \wedge\left\langle q_{h_{n-1} h_{n}}, q_{h_{n-1} h_{n}}^{\prime}\right\rangle\right)$

$$
\left\langle q_{i j}^{n}, q_{i j}^{n}\right\rangle \leq\left\langle q_{h_{0} h_{a}}^{a}, q_{h_{0} h_{a}}^{a}\right\rangle \wedge\left\langle q_{h_{a} h_{a}}^{b-a}, q_{h_{a} h_{a}}^{b-a}\right\rangle \wedge\left\langle q_{h_{a} h_{n}}^{n-b}, q_{h_{a} h_{n}}^{\prime n-b}\right\rangle
$$

where $1<b-a<n$.

There exists $t \in \overline{I^{+}}$such that $[n]=t(b-a)$,

Hence

$\left\langle q_{h_{a} h_{a}}^{[n]}, q_{h_{a} h_{a}}^{[[n]}\right\rangle \geq\left\langle q_{h_{a} h_{a}}^{b-a}, q_{h_{a} h_{a}}^{b-a}\right\rangle \wedge\left\langle q_{h_{a} h_{a}}^{b-a}, q_{h_{a} h_{a}}^{\prime b-a}\right\rangle \ldots \wedge\left\langle q_{h_{a} h_{a}}^{b-a}, q_{h_{a} h_{a}}^{\prime b-a}\right\rangle$ $=\left\langle q_{h_{a} h_{a}}^{b-a}, q_{h_{a} h_{a}}^{b-a}\right\rangle(\mathrm{t}$ terms$)$

and so

$\left\langle q_{i j}^{n}, q_{i j}^{\prime n}\right\rangle \leq\left\langle q_{h_{0} h_{a}}^{a}, q_{h_{0} h_{a}}^{\prime a}\right\rangle \wedge\left\langle q_{h_{a} h_{a}}^{b-a}, q_{h_{a} h_{a}}^{\prime b-a}\right\rangle \wedge\left\langle q_{h_{a} h_{a}}^{[n]}, q_{h_{a} h_{a}}^{\prime[n]}\right\rangle \wedge\left\langle q_{h_{a} h_{a}}^{n-b}, q_{h_{a} h_{a}}^{\prime n-b}\right\rangle$

$$
\leq\left\langle q_{h_{0} h_{n}}^{n+[n]}, q_{h_{0} h_{n}}^{\prime n+[n]}\right\rangle=\left\langle q_{i j}^{n+[n]}, q_{i j}^{\prime n+[n]}\right\rangle .
$$

By Theorem 3.3 and (2) we have $d^{\bullet} \mid[n]$, that is (1) holds.

Corollary 3.8. $1 \leq d^{\bullet} \leq[n]$

Theorem 3.9. If there exists a positive integer $h$ such that $\left\langle q_{i i}^{k}, q_{i i}^{\prime k}\right\rangle \leq\left\langle q_{i i}^{h}, q_{i i}^{\prime h}\right\rangle$ for all $i, j \in\{1,2, \ldots, n\}$, then we have

(1) $\left(\left\langle q_{i j}^{n}, q_{i j}^{\prime n}\right\rangle\right) \leq\left(\left\langle q_{i j}^{n+h}, q_{i j}^{\prime n+h}\right\rangle\right)$;

(2) $d^{\bullet} \mid h$;

(3) if $(h,[n])=1$, then $\left(\left\langle q_{i j}, q_{i j}^{\prime}\right\rangle\right)$ is convergent.

Proof. (1) By (10), we have

$\left\langle q_{i j}^{n}, q_{i j}^{\prime n}\right\rangle \leq\left\langle q_{i h_{a}}^{a}, q_{i h_{a}}^{\prime a}\right\rangle \wedge\left\langle q_{h_{a} h_{a}}^{b-a}, q_{h_{a} h_{a}}^{b-a}\right\rangle \wedge\left\langle q_{h_{a} j}^{n-b}, q_{h_{a j}}^{\prime n-b}\right\rangle$

$=\left\langle q_{i h_{a}}^{a}, q_{i h_{a}}^{\prime a}\right\rangle \wedge\left\langle q_{h_{a} h_{a}}^{b-a}, q_{h_{a} h_{a}}^{b-a}\right\rangle \wedge\left\langle q_{h_{a} h_{a}}^{h}, q_{h_{a} h_{a}}^{\prime h}\right\rangle \wedge\left\langle q_{h_{a} j}^{n-b}, q_{h_{a} j}^{\prime n-b}\right\rangle$

$\leq\left\langle q_{i j}^{n+h}, q_{i j}^{\prime n+h}\right\rangle$

and so $\left(\left\langle q_{i j}^{n}, q_{i j}^{\prime n}\right\rangle\right)$

$\leq\left(\left\langle q_{i j}^{n+h}, q_{i j}^{\prime n+h}\right\rangle\right)$

(2) By (1) and Theorem 3.3, we have $d^{\bullet} \mid h$.

(3) By Theorem 3.7 and (2), we get $d^{\bullet} \mid[n]$;

since $d^{\bullet} \mid h$ and thus $d^{\bullet} \mid 1$ we find $d^{\bullet}=1$.

$\Rightarrow\left(\left\langle q_{i j}, q_{i j}^{\prime}\right\rangle\right)$ is convergent.

Corollary 3.10. If $\left\langle q_{i i}^{k}, q_{i i}^{\prime k}\right\rangle \leq\left\langle q_{i i}^{n}, q_{i i}^{\prime n}\right\rangle$. for all $i, k \in\{1,2, \ldots, n\}$, then (1) $d^{\bullet} \mid n$ and (2) $\left(\left\langle q_{i j}^{n}, q_{i j}^{\prime n}\right\rangle\right) \leq\left(\left\langle q_{i i}^{2 n}, q_{i j}^{\prime 2 n}\right\rangle\right)$.

Corollary 3.11. If there exists a positive integer $h$ such that $\left\langle q_{i i}^{k}, q_{i i}^{\prime k}\right\rangle \leq\left\langle q_{i i}^{n}, q_{i i}^{\prime n}\right\rangle \leq\left\langle q_{i i}^{h}, q_{i i}^{\prime h}\right\rangle$ for all $i, k \in\{1,2, \ldots, n\}$, and $(n, h)=1$, then $\left(\left\langle q_{i j}, q_{i j}^{\prime}\right\rangle\right)$ is convergent.

Proof. By Corollary 3.10 and Theorem 3.9, we have $d^{\bullet} \mid n$, and $d^{\bullet} \mid h$. Thus $d^{\bullet} \mid 1$, and so $d^{\bullet}=1$.

\section{An algorithm to find the period of oscillation}

Let $\left(\left\langle q_{i j}, q_{i j}^{\prime}\right\rangle\right)$ be an $n \times n$ IFM, and $d^{\bullet}, k^{\bullet}$ be the period of oscillation and the index of $\left(\left\langle q_{i j}, q_{i j}^{\prime}\right\rangle\right)$, respectively.

We develop the following algorithm to find the $d^{\bullet}$ and $k^{\bullet}$. Step 1. Compute successively

$$
\left(\left(\left\langle q_{i j}, q_{i j}^{\prime}\right\rangle\right),\left(\left\langle q_{i j}^{1+[n]}, q_{i j}^{\prime 1+[n]}\right\rangle\right)\right),\left(\left(\left\langle q_{i j}^{2}, q_{i j}^{\prime 2}\right\rangle\right)\right.
$$

$$
\left.\left(\left\langle q_{i j}^{2+[n]}, q_{i j}^{2+[n]}\right\rangle\right)\right), \ldots,\left(\left(\left\langle q_{i j}^{m}, q_{i j}^{\prime m}\right\rangle\right),\left(\left\langle q_{i j}^{m+[n]}, q_{i j}^{\prime m+[n]}\right\rangle\right)\right), \ldots
$$


In (12) find $k$ such that

$$
\left(\left\langle q_{i j}^{k-1}, q_{i j}^{\prime k-1}\right\rangle\right) \neq\left(\left\langle q_{i j}^{(k-1)+[n]}, q_{i j}^{(k-1)+[n]}\right\rangle\right)
$$

but

$$
\left(\left\langle q_{i j}^{k}, q_{i j}^{\prime k}\right\rangle\right)=\left(\left\langle q_{i j}^{k+[n]}, q_{i j}^{\prime k+[n]}\right\rangle\right)
$$

Then $k^{\bullet}=k$. Go to Step 2 .

Step 2. Compute successively

$$
\begin{aligned}
& \left(\left\langle q_{i j}^{k^{\bullet}}, q_{i j}^{\prime k^{\bullet}}\right\rangle\right),\left(\left\langle q_{i j}^{k^{\bullet}+1}, q_{i j}^{\prime k^{\bullet}+1}\right\rangle\right),\left(\left\langle q_{i j}^{k^{\bullet}+2}, q_{i j}^{\prime k^{\bullet}+2}\right\rangle\right), \ldots, \\
& \left(\left\langle q_{i j}^{k^{\bullet}+[n]}, q_{i j}^{\prime k^{\bullet}[n]}\right\rangle\right)
\end{aligned}
$$

In (13) find $d$ such that

$$
\begin{aligned}
& \left(\left\langle q_{i j}^{k^{\bullet}}, q_{i j}^{\prime k^{\bullet}}\right\rangle\right) \neq\left(\left\langle q_{i j}^{\left(k^{\bullet}+j\right.}, q_{i j}^{\prime k^{\bullet}+j}\right\rangle\right) \text { for } j=1,2, \ldots, d-1 \\
& \operatorname{but}\left(\left\langle q_{i j}^{k^{\bullet}}, q_{i j}^{\prime k^{\bullet}}\right\rangle\right)=\left(\left\langle q_{i j}^{k^{\bullet}+d}, q_{i j}^{\prime k^{\bullet}+d}\right\rangle\right) .
\end{aligned}
$$

Then $d^{\bullet}=d$. Stop

Proof. Step 1. By Theorem 3.7 and Lemma 3.2, we have $\left(\left\langle q_{i j}^{k^{\bullet}}, q_{i j}^{\prime k^{\bullet}}\right\rangle\right)=\left(\left\langle q_{i j}^{k^{\bullet}+[n]}, q_{i j}^{\prime k^{\bullet}+[n]}\right\rangle\right)$.

Hence, the integer $k$ does exist.

Since $\left(\left\langle q_{i j}^{k-1}, q_{i j}^{\prime k-1}\right\rangle\right) \neq\left(\left\langle q_{i j}^{(k-1)+[n]}, q_{i j}^{\prime(k-1)+[n]}\right\rangle\right)$, then by Theorem 3.5, and Theorem 3.7, we get $k-1<k^{\bullet}$, and $k \leq k^{\bullet}$. Since $\left(\left\langle q_{i j}^{k}, q_{i j}^{\prime k}\right\rangle\right) \neq\left(\left\langle q_{i j}^{(k)+[n]}, q_{i j}^{\prime(k)+[n]}\right\rangle\right)$, using Theorem 3.5 again, we have $k \geq k^{\bullet}$, so $k=k^{\bullet}$.

Step 2. From the fact that $\left(\left\langle q_{i j}^{k^{\bullet}}, q_{i j}^{\prime k^{\bullet}}\right\rangle\right)=\left(\left\langle q_{i j}^{k^{\bullet}}+d^{\bullet}, q_{i j}^{\prime k^{\bullet}}+d^{\bullet}\right\rangle\right)$, we know the integer $d$ does not exist. Since $\left(\left\langle q_{i j}^{k^{\bullet}}, q_{i j}^{\prime k^{\bullet}}\right\rangle \neq\right.$ $\left(\left\langle q_{i j}^{k^{\bullet}+j}, q_{i j}^{\prime k^{\bullet}+j}\right\rangle\right)$ for $j=1,2, . ., d-1$, then by the definition of $d^{\bullet}$, we have $d^{\bullet}>d-1$.

Therefore, $d^{\bullet} \geq d$. But $\left(\left\langle q_{i j}^{k^{\bullet}}, q_{i j}^{\prime k^{\bullet}}\right\rangle\right)=\left(\left\langle q_{i j}^{k^{\bullet}+d}, q_{i j}^{\prime k^{\bullet}}+d\right\rangle\right)$ and by Theorem 3.5, we have $d^{\bullet} \leq d$. So, $d^{\bullet}=d$

Example 4.1. Here, $[n]=[3]=6$.

$$
\begin{gathered}
Q=\left[\begin{array}{lll}
\langle 0.2,0.7\rangle & \langle 0.3,0.6\rangle & \langle 0.6,0.3\rangle \\
\langle 0.8,0.1\rangle & \langle 0.0,1.0\rangle & \langle 0.5,0.4\rangle \\
\langle 0.4,0.5\rangle & \langle 0.7,0.2\rangle & \langle 0.1,0.8\rangle
\end{array}\right] \\
Q^{2}=\left[\begin{array}{lll}
\langle 0.4,0.5\rangle & \langle 0.6,0.3\rangle & \langle 0.3,0.6\rangle \\
\langle 0.4,0.5\rangle & \langle 0.5,0.4\rangle & \langle 0.6,0.3\rangle \\
\langle 0.7,0.2\rangle & \langle 0.3,0.6\rangle & \langle 0.5,0.4\rangle
\end{array}\right] \\
\neq\left[\begin{array}{lll}
\langle 0.6,0.3\rangle & \langle 0.5,0.4\rangle & \langle 0.5,0.4\rangle \\
\langle 0.5,0.4\rangle & \langle 0.6,0.3\rangle & \langle 0.5,0.4\rangle \\
\langle 0.5,0.4\rangle & \langle 0.5,0.4\rangle & \langle 0.6,0.3\rangle
\end{array}\right]=Q^{2+[3]}=Q^{6}
\end{gathered}
$$

$$
\begin{gathered}
Q^{3}=\left[\begin{array}{lll}
\langle 0.6,0.3\rangle & \langle 0.3,0.6\rangle & \langle 0.5,0.4\rangle \\
\langle 0.5,0.4\rangle & \langle 0.6,0.3\rangle & \langle 0.5,0.4\rangle \\
\langle 0.4,0.5\rangle & \langle 0.5,0.4\rangle & \langle 0.6,0.3\rangle
\end{array}\right] \\
\neq\left[\begin{array}{lll}
\langle 0.6,0.3\rangle & \langle 0.5,0.4\rangle & \langle 0.5,0.4\rangle \\
\langle 0.5,0.4\rangle & \langle 0.6,0.3\rangle & \langle 0.5,0.4\rangle \\
\langle 0.5,0.4\rangle & \langle 0.5,0.4\rangle & \langle 0.6,0.3\rangle
\end{array}\right]=Q^{3+[3]}=Q^{9} \\
Q^{4}=\left[\begin{array}{lll}
\langle 0.4,0.5\rangle & \langle 0.5,0.4\rangle & \langle 0.6,0.3\rangle \\
\langle 0.6,0.3\rangle & \langle 0.5,0.4\rangle & \langle 0.5,0.4\rangle \\
\langle 0.5,0.4\rangle & \langle 0.6,0.3\rangle & \langle 0.5,0.4\rangle
\end{array}\right] \\
=\left[\begin{array}{lll}
\langle 0.5,0.4\rangle & \langle 0.5,0.4\rangle & \langle 0.6,0.3\rangle \\
\langle 0.6,0.3\rangle & \langle 0.5,0.4\rangle & \langle 0.5,0.4\rangle \\
\langle 0.5,0.4\rangle & \langle 0.6,0.3\rangle & \langle 0.5,0.4\rangle
\end{array}\right]=Q^{3+[3]}=Q^{10} \\
Q^{5}=\left[\begin{array}{lll}
\langle 0.5,0.4\rangle & \langle 0.6,0.3\rangle & \langle 0.5,0.4\rangle \\
\langle 0.5,0.4\rangle & \langle 0.5,0.4\rangle & \langle 0.6,0.3\rangle \\
\langle 0.6,0.3\rangle & \langle 0.5,0.4\rangle & \langle 0.5,0.4\rangle
\end{array}\right] \\
=\left[\begin{array}{lll}
\langle 0.5,0.4\rangle & \langle 0.6,0.3\rangle & \langle 0.5,0.4\rangle \\
\langle 0.5,0.4\rangle & \langle 0.5,0.4\rangle & \langle 0.6,0.3\rangle \\
\langle 0.6,0.3\rangle & \langle 0.5,0.4\rangle & \langle 0.5,0.4\rangle
\end{array}\right]=Q^{3+[3]}=Q^{11} .
\end{gathered}
$$

So, $k^{\bullet}=5$. Further,

$$
\begin{gathered}
Q^{5}=\left[\begin{array}{lll}
\langle 0.5,0.4\rangle & \langle 0.6,0.3\rangle & \langle 0.5,0.4\rangle \\
\langle 0.5,0.4\rangle & \langle 0.5,0.4\rangle & \langle 0.6,0.3\rangle \\
\langle 0.6,0.3\rangle & \langle 0.5,0.4\rangle & \langle 0.5,0.4\rangle
\end{array}\right] \\
\neq=\left[\begin{array}{lll}
\langle 0.6,0.3\rangle & \langle 0.5,0.4\rangle & \langle 0.5,0.4\rangle \\
\langle 0.5,0.4\rangle & \langle 0.6,0.3\rangle & \langle 0.5,0.4\rangle \\
\langle 0.5,0.4\rangle & \langle 0.5,0.4\rangle & \langle 0.6,0.3\rangle
\end{array}\right]=Q^{5+1}=Q^{6} . \\
Q^{5}=\left[\begin{array}{lll}
\langle 0.5,0.4\rangle & \langle 0.6,0.3\rangle & \langle 0.5,0.4\rangle \\
\langle 0.5,0.4\rangle & \langle 0.5,0.4\rangle & \langle 0.6,0.3\rangle \\
\langle 0.6,0.3\rangle & \langle 0.5,0.4\rangle & \langle 0.5,0.4\rangle
\end{array}\right] \\
=\left[\begin{array}{lll}
\langle 0.5,0.4\rangle & \langle 0.5,0.4\rangle & \langle 0.6,0.3\rangle \\
\langle 0.6,0.3\rangle & \langle 0.5,0.4\rangle & \langle 0.5,0.4\rangle \\
\langle 0.5,0.4\rangle & \langle 0.6,0.3\rangle & \langle 0.5,0.4\rangle
\end{array}\right]=Q^{5+2}=Q^{7}, \\
\left.b u t, \quad \begin{array}{lll}
\langle 0.5,0.4\rangle & \langle 0.6,0.3\rangle & \langle 0.5,0.4\rangle \\
\langle 0.5,0.4\rangle & \langle 0.5,0.4\rangle & \langle 0.6,0.3\rangle \\
\langle 0.6,0.3\rangle & \langle 0.5,0.4\rangle & \langle 0.5,0.4\rangle
\end{array}\right] \\
Q^{5}=\left[\begin{array}{lll}
\langle 0.5,0.4\rangle & \langle 0.6,0.3\rangle & \langle 0.5,0.4\rangle \\
\langle 0.5,0.4\rangle & \langle 0.5,0.4\rangle & \langle 0.6,0.3\rangle \\
\langle 0.6,0.3\rangle & \langle 0.5,0.4\rangle & \langle 0.5,0.4\rangle
\end{array}\right]=Q^{5+3}=Q^{8} .
\end{gathered}
$$$$
\text { So, } d^{\bullet}=3
$$

\section{Conclusion}

In this paper, we considered an arbitrary square IFM and defined its period of oscillation $d^{\bullet}$ and index $k^{\bullet}$. Moreover, we have developed some properties of $d^{\bullet}$ and $k^{\bullet}$ and proved that $d^{\bullet} \mid[n]$ (where $[n]$ is the least common multiple of $1,2, \ldots, \mathrm{n}$ ). Also, we have given the algorithm to find $d^{\bullet}$ and $k^{\bullet}$.

\section{References}

[1] Adak. A. K, Bhowmik. M and Pal. M. (2011). Application of generalized intuitionistic fuzzy matrix in multi-criteria decision making problem, Journal of Mathematical and Computational Science, 1 (1): 19-31. 
[2] Adak. A. K, Bhowmik. Mand Pal. M. (2012) Some properties of generalized intuitionistic fuzzy nilpotent matrices and its some properties, International Journal of Fuzzy Information and Engineering, 4: 371-387.

[3] Atanassov, K. (1987) Generalized index matrices, C. R. Acad Bulgare Sci. 40(11): 15-18.

[4] Atanassov, K. (1994) Index matrix representation of the intuitionistic fuzzy garphs, Proceeding Of Fifth Scientific Session Of The Math Foundation Of Artificial Intelligence Seminar, Sofia, 36-41. Accessed 5 Oct 1994 (Preprint MRL-MFAIS-10-94).

[5] Atanassov, K. (1983). Intuitionistic fuzzy sets. VII ITKR's Session, Sofia, June.

[6] Atanassov, K. (1999). Intuitionistic fuzzy sets ; Theory and Applications. Physica Verlag.

[7] Atanassov, K. (2005). Intuitionistic fuzzy implications and modus ponens. Notes on Intuitionistic Fuzzy Sets, (11)(1): 1-5.

[8] Atanassov, K. (2005). On some types of fuzzy negations. Notes on Intuitionistic Fuzzy Sets, (11)(4):170-172.

[9] Atanassov, K. (2005). A new intuitionistic fuzzy implication from a modal type. Advance Studies in Contemporary Mathematics, (12)(1): 117-122.

[10] Atanassov, K. and Gargov, G. (1998). Elements of intuitionistic fuzzy logic.part I., Fuzzy Sets and Systems, (95): 39-52.

[11] Atanassov, K. 2014 Index matrices towards an augmented matrix calculus, Springer, Cham.

[12] Bhowmik, M. and Pal, M. (2008). Some results on intuitionistic fuzzy matrices and circulant intuitionistic fuzzy matrices. International Journal of Mathematical Sciences, 7(1-2): 81-96.

[13] Bhowmik, M. and Pal, M. (2008). Generalized intuitionistic fuzzy matrices. Far East Journal of Mathematical Sciences, 29(3): 533-554.

[14] Im, Y, B. (2006) The determinant of square intuitionistic fuzzy matrices. Acta Ciencia Indica, XXXII M: 515-524.

[15] Lee. H. Y and Jeong. N. G. (2005). Canonical form of a transitive intuitionistic fuzzy matrices, Honam Mathematical Journal, 27 (4): 543-550.

[16] Meenakshi, A. R. and Gandhimathi, T. (2010). Intuitionistic fuzzy relational equations. advances in Fuzzy Mathematics, 5 (3): 239-244.

[17] Meenakshi, A. R. (2008). Fuzzy Matrix Theory And Applications. MJP Publishers, Chennai.

[18] Mondal. S and Pal. M. (2013). Similarity relations, invertibility and eigenvalues of intuitionistic fuzzy matrix, International Journal of Fuzzy Information and Engineering, 4: 431-443

[19] Mondal. S and Pal. M. (2014) Intuitionistic fuzzy incline matrix and determinant, Annals of Fuzzy Mathematics and Informatics, 8(1): 19-32.

[20] Murugadas. P and Padder R. A. (2015) Reduction of an intuitionistic fuzzy rectangular Matrix, Annamalai University Science Journal, 49: 15-18.
[21] Ovchinnikov S. V.(1981), Structure of fuzzy binary relations, Fuzzy Sets and Systems 6: 169-195.

[22] Padder R. A and Murugadas. P.Max-max operation on intuitionistic fuzzy matrix, Annals of Fuzzy Mathematics and Informatics, Article in press.

[23] Padder R. A and Murugadas. P. Convergence of powers and canonical form of s-transitive intuitionistic fuzzy matrix New Trends in Mathematical Sciences, Article in press.

[24] Pal, M., Khan, S. k. and A. K Shyamal. (2002). Intuitionistic fuzzy matrices. Notes on Intuitionistic Fuzzy Sets, 8 (2): 51-62.

[25] Pradhan. R and Pal. M. (2013) Convergence of maxarithmetic mean-minarithmetic mean powers of intuitionistic fuzzy matrices, intern. J. Fuzzy Mathematical Archive, 2: 58-69.

[26] Pradhan. R and Pal. M. (2012) Intuitionistic fuzzy linear transformations, Annals of pure and applied Mathematics, 1(1): 57-68.

[27] Pradhan. R and Pal. M. (2014) The generalized inverse of Atanassov's intuitionistic fuzzy matrices, International Journal of Computational Intelligence Systems, 7(6): 1083-1095.

[28] Shyamal, A.K. and Pal. M. (2002). Distances between intuitionistic fuzzy matrices. V.U.J. Physical Sciences, 8: 81-91.

[29] Sriram, S. and Murugadas, P. (2011). Sub-inverses of intuitionistic fuzzy matrices. Acta Ciencia Indica (Mathematics), Vol.XXXVII, M N0 1: 41-56.

[30] Sriram, S. and Murugadas, P. (2010). On semi-ring of intuitionistic fuzzy matrices. Applied Mathematical Science, 4(23): 1099-1105.

[31] Xu, Z. and Yager, R.R. (2006). Some geometric operators based on Intuitionistic Fuzzy Sets. Int. Journal of General Systems, 35: 417-433.

[32] Zadeh. L.A. (1965). Fuzzy Sets, Journal of Information and Control, 8: 338-353.

[33] R.H. Kim and F.W. Roush, Generalized fuzzy matrices, Fuzzy Sets and Systems, 4, (1980) 293-315.

[34] M. G Thomason, Convergence of powers of a fuzzy matrix, J. Math. Anal. Appl. 57(1977) 476-480.

[35] Z. T Ran and D. F. Liu, On the oscillating power sequence of a fuzzy matrix, Fuzzy sets and systems, 93 (1998) 7585.

[36] Y. Y. Lur, Y. K.Wu and S. M. Guu, Convergence of maxarithmetic mean power of a fuzzy matrix, Fuzzy sets and system, 158 (2007) 2516-2522.

[37] H.Hashimoto, Convergence of powers of a fuzzy transitive matrix, Fuzzy sets and systems, 9 (1983) 153-160.

[38] D. A. Gregory, S.Kirkland and N.J Pullman, Power convergent boolean matrices, Linear algebra and its applications, 179 (1993) 105-117.

[39] J. J. Buckley, Note on convergence of powers of a fuzzy matrix, Fuzzy sets and systems, 121 (2001) 363-364.

[40] Sa Dan, Wu Wangming, Graphics characteristics of 
power convergence of fuzzy matrix, Fuzzy Math. 1 (1987) $1-4$ (in Chinese).

[41] Z.-T. Fan, D.-F. Liu, On the oscillating power sequence of a fuzzy matrix, Fuzzy Sets and Systems 93 (1998) 75-85.

[42] J.-X. Li, Controllable fuzzy matrices, Fuzzy Sets and Systems 45 (3) (1992) 313-319.

[43] J.-X. Li, Periodicity of powers of fuzzy matrices, Fuzzy Sets and Systems 48 (1992) 365-369.

[44] J.-X. Li, An upper bound on indices of infinite fuzzy relations, Fuzzy Sets and Systems 49 (1992) 317-321.

[45] J.-X. Li, Convergence of powers of controllable fuzzy matrices, Fuzzy Sets and Systems 62 (1) (1994) 83-88.

[46] W. Liua, Z. Ji, , Fuzzy Sets and Systems 126 (1) (2002) 233-240.

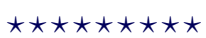

ISSN(P):2319- 3786

Malaya Journal of Matematik

$\operatorname{ISSN}(\mathrm{O}): 2321-5666$

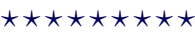

